\title{
3-D Elasticity-based Modeling of Anisotropic Piezocomposite Transducers for Guided Wave Structural Health Monitoring
}

\author{
Ajay Raghavan ${ }^{*}$ and Carlos E.S. Cesnik ${ }^{\dagger}$ \\ Department of Aerospace Engineering, University of Michigan, Ann Arbor, Michigan, 48109-2140
}

\begin{abstract}
Anisotropic piezocomposite transducers (APTs), such as macro fiber composites and active fiber composites, have great potential to be used as structurally integrated transducers for guided-wave structural health monitoring (GW SHM). Their main advantages over conventional monolithic piezoceramic wafer transducers are mechanical flexibility, curved surface conformability, power efficiency, their ability to excite focused GW fields, and their unidirectional sensing capability as a GW sensor. In this paper, models are developed to describe excitation of GW fields by 3-3 APT transducers in isotropic structures. The configurations explored are plane Lamb-wave fields in beams with rectangular cross-section, axisymmetric GW fields in cylinders, and 3-D GW fields in plates The dynamics of the substrate and transducer are assumed uncoupled. The actuator is modeled as causing shear traction at edges of the actuator's active area along the fiber direction. The sensor is modeled as sensing the average extensional strain over the active area along the fiber direction. The work is unique in that the formulation is based on 3-D elasticity, and no reduced order structural assumptions are used. This is crucial to model multimodal GW propagation, especially at high frequencies. A formulation is also proposed to model their behavior as GW sensors. Finally, results from experimental tests to examine the validity of the models are discussed and the possible sources of error examined in detail.
\end{abstract}

\section{Nomenclature}

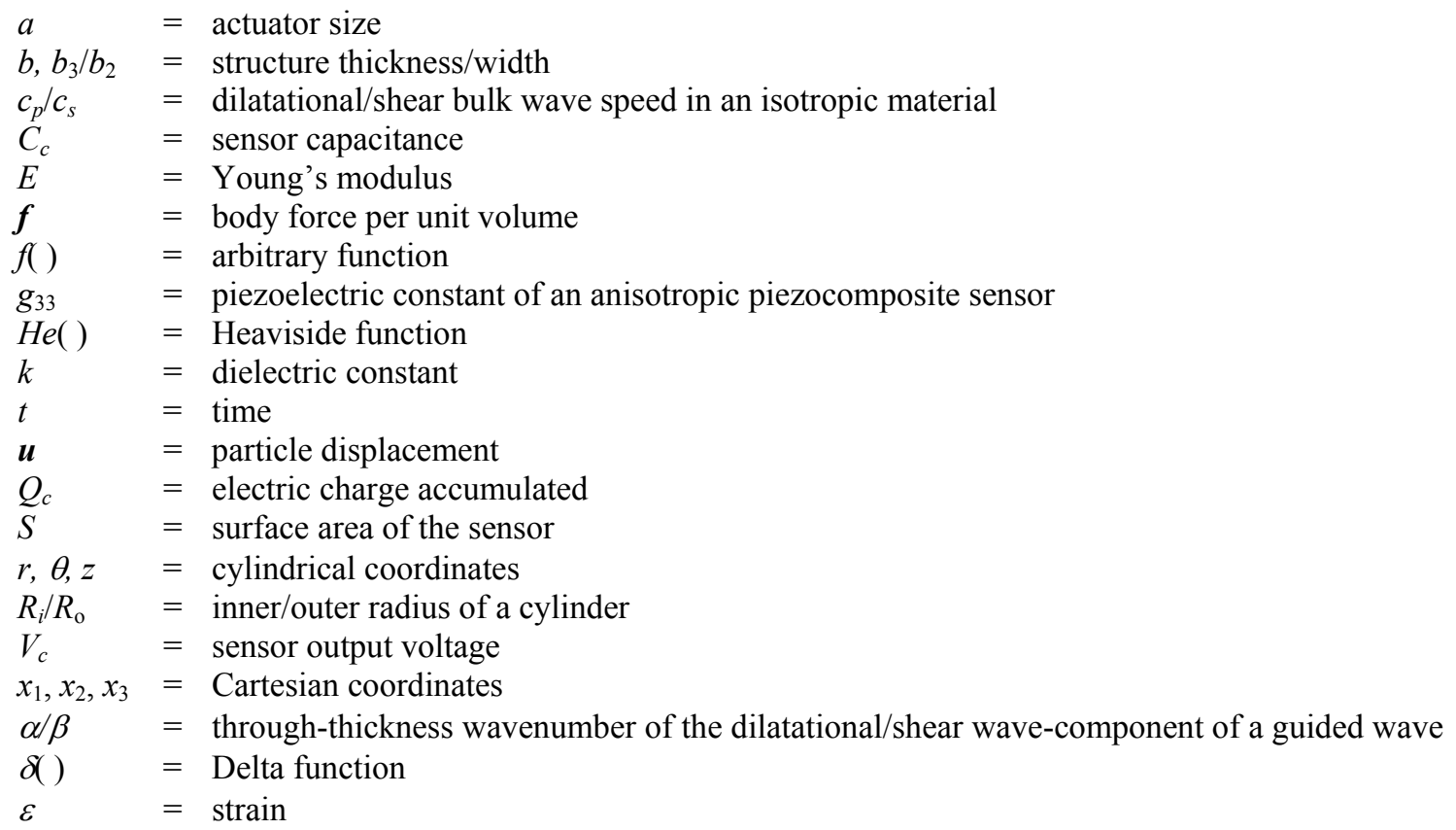

\footnotetext{
${ }^{*}$ Graduate Research Assistant and AIAA Member

† Associate Professor and Associate Fellow, AIAA; Corresponding author; Email: cesnik@umich.edu; Phone: 1734-764-3397; Fax: 1-734-763-0578
} 


$\begin{array}{ll}\varepsilon_{0} & =\text { electric permittivity of free space } \\ \phi & =\text { Helmholtz scalar potential } \\ \mathrm{H} & =\text { Helmholtz vector potential } \\ \varphi() & =\text { generic function used for definition of 2-D spatial Fourier transform } \\ \lambda, \mu & =\text { Lamé's constants for an isotropic material } \\ \rho & =\text { material density } \\ \sigma_{i j} & =\text { stress component }(i, j=1,2,3 \text { or } r, \theta, z) \\ \tau_{0} & =\text { magnitude of shear stress exerted by a 3-3 anisotropic piezocomposite actuator on its host structure } \\ v & =\text { Poisson's ratio } \\ \omega & =\text { angular frequency } \\ \xi & =\text { wavenumber of a propagating guided wave } \\ \psi & =\text { generic variable }\end{array}$

\section{Introduction}

$\mathrm{G}$ uided wave structural health monitoring (GW SHM) stands out as a highly promising solution for the important problem of damage prognosis in aerospace and mechanical structures. It essentially involves exciting GWs in the structure with high frequency tonebursts using structurally integrated transducers and analyzing the signals of the waves in the structure to extract information about damage in it, if present ${ }^{1}$. Most commonly, uniformly poled piezoceramic (typically lead zirconium titanate, i.e., PZT) wafer transducers are used to excite and sense GWs in this approach. However, these are ceramic, and hence rather brittle, making them very susceptible to damage during handling or in operation. For SHM, it is crucial to have transducers that can survive events causing damage to the structure and estimate the remaining service life of the structure in the aftermath of such events. In addition,
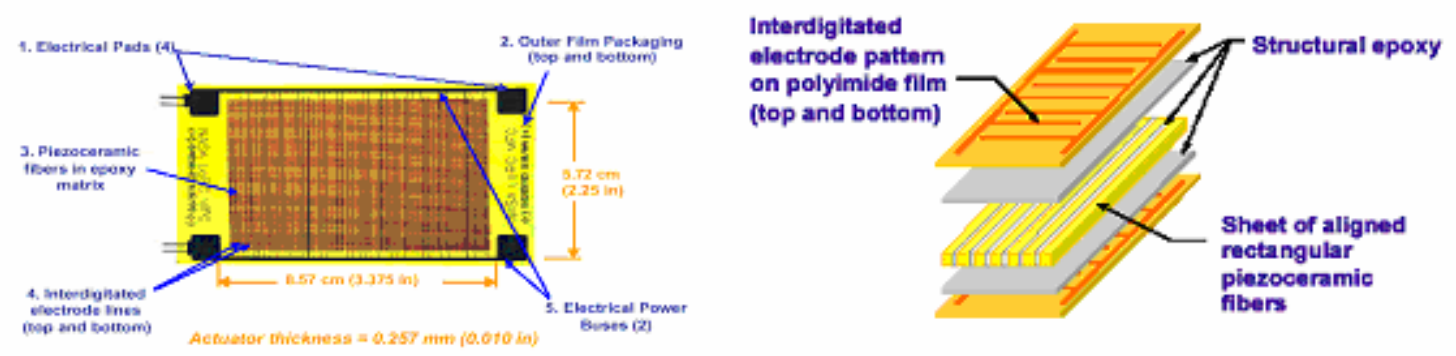

Fig. 1. a) (left) The packaged MFC and b) (right) The components of a MFC actuator ${ }^{2}$

piezoceramics have poor surface conformability and are not suitable for mounting on curved structures such as aircraft shells. In order to overcome these disadvantages of piezoceramics, various options have been explored. Monkhouse et al. $^{3}$ designed polyvinylidene fluoride (PVDF) films with copper backing layers to improve its response characteristics. An interdigitated electrode pattern was deposited using printed circuit board (PCB) techniques for modal selectivity. Badcock and Birt ${ }^{4}$ used PZT powder incorporated into an epoxy resin (base material) to form poled film sheets, which were used as transducer elements for GW generation and sensing. Hayward et al. ${ }^{5}$ designed interdigitated transducers (IDTs) with 1-3 coupling piezocomposite layers, consisting of modified lead titanate ceramic platelets held together by a passive soft-set epoxy polymer, and sandwiched between two PCBs for wavenumber and modal selectivity. However, the actuation capability of these alternatives was demonstrated to be poor compared to that of conventional uniformly poled piezoceramics.

Conformal anisotropic macro fiber composite (MFC) transducers, shown in Fig. 1, were developed at NASA Langley $^{2}$. They utilize interdigitated electrode poling and piezoceramic fibers embedded in an epoxy matrix. Fine ceramic fibers provide increased specific strength over monolithic materials, allowing conformability to curved surfaces. Compositing the ceramic provides alternate load path redundancy, increasing robustness to damage. It was shown that these types of actuators have significantly higher energy densities than monolithic piezoceramics in planar actuation for quasi-static applications ${ }^{6}$. In MFCs, by using the piezoelectric "3-3" mode of actuation along the fiber direction (instead of the transverse ones, like in the conventional piezoceramic wafer), the actuation authority 
can be theoretically as higher as three times that of a monolithic crystal (since the "3-3" piezoelectric constant is typically three times larger than the "3-1" piezoelectric constant). In addition, when used as a sensor, the more powerful converse effect causes its response to be stronger than that of a monolithic crystal (again, roughly by three times). Thus, MFCs provide the added advantage of being power efficient. Furthermore, due to the orientation of fibers along a particular direction, MFCs can be used to excite directionally focused GW fields in structures, as well as be insensitive to GWs incident normal to the fiber direction as sensors. Finally, by suitably tailoring their interdigitated electrode pattern, they can be tuned to excite particular wavelengths, and thereby achieve GW modal selectivity. All these characteristics make MFCs very attractive candidates for use as permanently mounted GW SHM transducers. MFCs and active fiber composites (AFCs), which are very similar in construction (with some differences in the fiber manufacturing process), have been explored by some researchers for use as GW SHM transducers in some preliminary tests with encouraging results ${ }^{7-10}$. The present paper addresses modeling of the GW field excited by these transducers and their response when used as GW sensors. These transducers are referred to as 3-3 anisotropic piezocomposite transducers (APTs) in this work.

The free GW modes in isotropic plates and cylinders were first studied by Lamb ${ }^{11}$ and Gazis ${ }^{12}$ respectively using the theory of elasticity. Earlier works on modeling excitation of GW fields using the theory of elasticity have mostly used 2-D plane wave models, wherein variations normal to the direction of propagation (but not through the structure thickness) were neglected. The book by Viktorov ${ }^{13}$ on 2-D models based on the theory of elasticity for Lamb wave excitation in isotropic plates by non-destructive testing (NDT) transducers was the pioneering work in this direction. A heuristic model was also proposed for extending the 2-D model to the case of 3-D excitation by NDE transducers. Ditri and Rose $^{14}$ used plane strain models to describe excitation in composites by NDT transducers. Santosa and $\mathrm{Pao}^{15}$ solved the generic 3-D problem of excitation of GWs in isotropic plate by an impulse point body force using the normal modes expansion technique. Wilcox ${ }^{16}$ presented a 3-D elasticity model describing the harmonic GW field by generic surface point sources in isotropic plates, however the model was not rigorously developed, and some intuitive reasoning was used to extend 2-D model results to 3-D. Giurgiutiu ${ }^{17}$ developed a 2-D model for plane Lamb-wave excitation by surface bonded piezo-actuators on isotropic plates. As pointed out in Ref. 17, the key difference between NDT transducers and surface-bonded piezo-transducers is that the former, not being permanently mounted on the structure, principally operate by "tapping" or causing normal traction on the free surface of the structure while the latter cause shear tractions. Some works have described the excitation of GW fields by finite dimension monolithic piezo-actuators using Mindlin plate theory ${ }^{18-20}$, but these can at best model one mode of the several GW modes that can be excited, and that too approximately. The only efforts in the literature that sought to address the modeling of anisotropic piezocomposite actuators for GW SHM were the works by Schulz et al. $^{7}$ and Datta et al. ${ }^{8}$. There, simple reduced order beam and plate models were presented to model GW excitation by AFCs in isotropic structures, without any experimental validation provided for the models. It is well known that at the high frequencies used for GW SHM, reduced order models are inadequate, being primarily developed for quasistatic to low frequency applications. Hence, there is a need to develop high fidelity theoretical models to model GW excitation and sensing in structures using 3-3 APTs, which will be very useful to gain insights into the nature of the GW field excited by these elements as well as to design GW SHM systems using these.

With the above in mind, this work aims to develop 3-D elasticity models for GW excitation and sensing by 3-3 APTs and test these models experimentally using MFCs.

\section{Theoretical Formulation}

Earlier work by the authors ${ }^{21-23}$ addressed excitation and sensing of GW fields by arbitrary shape finite dimension monolithic piezoelectric wafer transducers using 3-D elasticity. The actuator was modeled as causing shear traction on the structural substrate surface along the free edge of the actuator, in the direction normal to the free edge. The sensor was modeled as sensing the average in-plane extensional surface strain over its bonded surface area. Thus, effectively the dynamics of the transducer and the plate are assumed uncoupled, and this was theoretically and experimentally shown to be a good assumption when the product of the transducer stiffness and thickness was small compared to that of the plate. To the best knowledge of the authors, this is the first work in the literature to provide $\mathrm{GW}$ field solutions for finite dimension piezo-actuators using 3-D elasticity. The present work seeks to develop and verify analogous models based on the theory of elasticity for 3-3 APTs in various structural configurations. It is hypothesized that a similar approach can be used to model GW excitation and sensing by finite dimension 3-3 APTs. The key difference is that the 3-3 APT, when used as an actuator, is modeled as causing shear traction at the edges of the active area (the surface area occupied by piezoelectric fibers) along the fiber direction only. Similarly, when it is used as a sensor, it is modeled as only being sensitive to the in-plane extensional strain component along the fiber direction, again over the active area. For modeling the excitation when used as actuator, 
the GW field solution in terms of displacement and strain was obtained by solving the 3-D elasticity-based boundary value problem using integral spatial transforms and the residue theorem from complex analysis. As in the earlier work by the authors ${ }^{21-23}$ on modeling monolithic piezoelectric transducers, two important assumptions are made in the subsequent theoretical analysis. One is that the frequency dependence of the piezoelectric constants is negligible, which is a reasonable assumption in lieu of the work by González and Alemany ${ }^{24}$. The other is that material damping can be ignored. This is also a practical assumption for metallic structures, since damping tends to be negligible in metals. Models are developed for the cases of plane Lamb-wave excitation in isotropic beams, axisymmetric GW excitation in isotropic cylinders and 3-D GW excitation in plates by surface-bonded 3-3 APTs. A generic formulation for the voltage response of the 3-3 APT when used as a sensor is also developed.

\section{A. Plane Lamb-wave Excitation in Isotropic Beams with Rectangular Cross-Section}

For the case of plane Lamb-wave excitation by 3-3 APTs in isotropic beams with a uniform rectangular crosssection, the configuration for which a solution is sought is shown in Fig. 2. The beam is infinitely long along the $x_{1}-$ direction and the other two dimensions are $2 b_{2}$ and $2 b_{3}$ respectively along the $x_{2}$ and $x_{3}$-directions. The 3-3 APT spans or nearly spans the width of the beam on one free surface. Its fibers are oriented along the beam axis, i.e., the $x_{1}$-direction. If the problem is defined rigorously, i.e., if traction free surface conditions are to be satisfied along all the four sides of the cross-section, in principle the solution consists of an infinite number of modes at any frequency

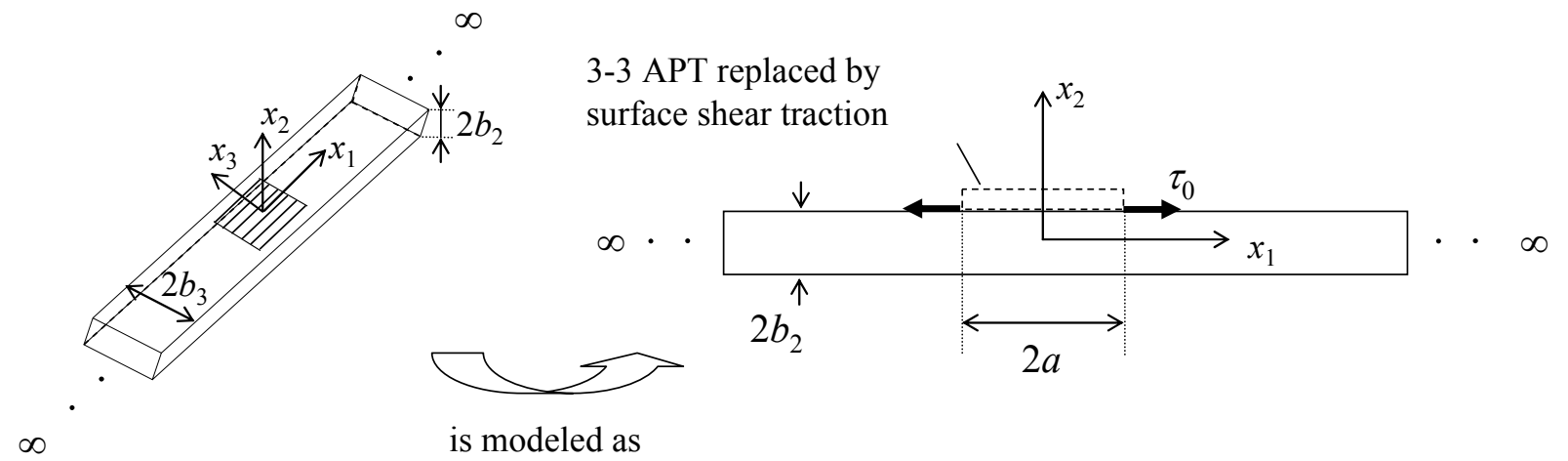

Fig. 2. Model for 3-3 APTs surface-bonded on isotropic beams with rectangular cross-section

and the problem is very complex to solve analytically. More details on such an approach can be found in Kastrzhitskaya and Meleshko ${ }^{25}$. However, if $2 b_{3}>2 b_{2}$, then the configuration can be approximated to be a 2-D plane wave problem along the beam axis, and the condition $\frac{\partial}{\partial \boldsymbol{x}_{3}}()=0$ can be used to simplify the governing equations. The derivation by Giurgiutiu ${ }^{17}$ for 2-D models describing Lamb-wave excitation by piezoceramics is then applicable here. As discussed above, the effect of the 3-3 APT is to cause shear tractions at $\left(a, b_{2}\right)$ and $\left(-a, b_{2}\right)$ as shown. Thus, one obtains the following surface condition (for harmonic excitation at angular frequency $\omega$ ):

$$
\sigma_{21}\left(x_{2}=b\right)=\tau_{0}\left[\delta\left(x_{1}-a\right)-\delta\left(x_{1}+a\right)\right] e^{-i \omega t}
$$

where $\tau_{0}$ is the magnitude of the shear traction. To solve the boundary value problem, the 1-D spatial Fourier transform is applied along the $x_{1}$-direction and after applying the surface conditions along $x_{2}=b$ and $x_{2}=-b$, the following expression is obtained for displacement along the $x_{1}$-direction (the residue theorem from complex analysis is used for the Fourier spatial inversion):

$$
u_{1}\left(x_{1}, x_{2}=+b, t\right)=-\frac{i \tau_{0}}{\mu} \sum_{\xi^{S}} \frac{\sin \xi^{S} a}{\xi^{S}} \frac{N_{S}\left(\xi^{S}\right)}{D_{S}^{\prime}\left(\xi^{S}\right)} e^{i\left(\xi^{S} x_{1}-\omega t\right)}-\frac{i \tau_{0}}{\mu} \sum_{\xi^{A}} \frac{\sin \xi^{A} a}{\xi^{A}} \frac{N_{A}\left(\xi^{A}\right)}{D_{A}^{\prime}\left(\xi^{A}\right)} e^{i\left(\xi^{A} x_{1}-\omega t\right)}
$$

where 


$$
\begin{aligned}
& \alpha^{2}=\frac{\omega^{2}}{c_{1}^{2}}-\xi^{2} ; \beta^{2}=\frac{\omega^{2}}{c_{2}^{2}}-\xi^{2} ; c_{p}=\sqrt{\frac{\lambda+2 \mu}{\rho}} ; c_{s}=\sqrt{\frac{\mu}{\rho}} \\
& N_{S}=\xi \beta\left(\xi^{2}+\beta^{2}\right) \cos \alpha b \cos \beta b ; D_{S}=\left(\xi^{2}-\beta^{2}\right)^{2} \cos \alpha b \sin \beta b+4 \xi^{2} \alpha \beta \sin \alpha b \cos \beta b \\
& N_{A}=\xi \beta\left(\xi^{2}+\beta^{2}\right) \sin \alpha b \sin \beta b ; D_{A}=\left(\xi^{2}-\beta^{2}\right)^{2} \sin \alpha b \cos \beta b+4 \xi^{2} \alpha \beta \cos \alpha b \sin \beta b
\end{aligned}
$$

$\lambda$ and $\mu$ are Lamé's constants for the plate material and $\rho$ is the material density. The superscript $S$ corresponds to the symmetric Lamb modes and the superscript $A$ corresponds to the antisymmetric Lamb modes. The wavenumber $\xi$ of a specific mode for a given $\omega$ is obtained from the solutions of the Rayleigh-Lamb equation for free waves in an isotropic plate, which is:

$$
\frac{\tan \beta b}{\tan \alpha b}=\left[\frac{-4 \alpha \beta \xi^{2}}{\left(\xi^{2}-\beta^{2}\right)^{2}}\right]^{ \pm 1}
$$

where the positive exponent is for symmetric Lamb modes and the negative one is for antisymmetric Lamb modes. The dispersion curves (plots of phase velocity $c=\omega / \xi$ versus harmonic frequency) for the Lamb modes in Aluminum are shown in Fig. 3. While harmonic excitation is considered here and the subsequent sections, the response to any excitation signal can be obtained by taking the inverse Fourier transform over the excited frequency range.

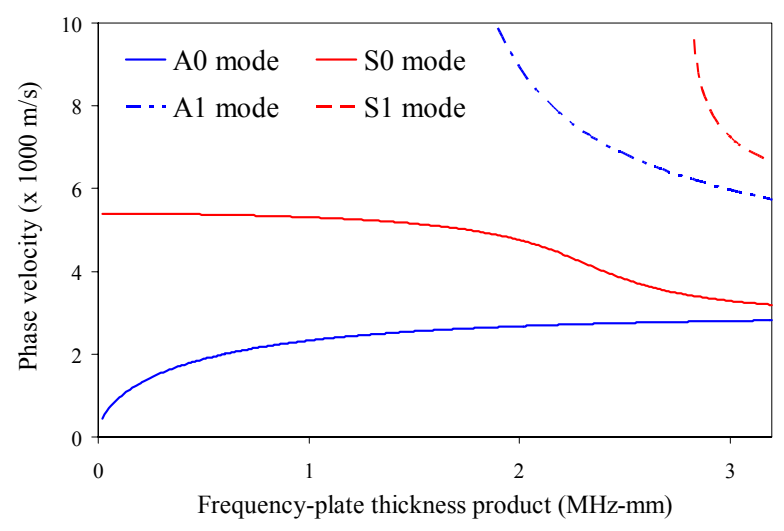

Fig. 3. Phase velocity dispersion curves for the Lamb modes in an Aluminum plate

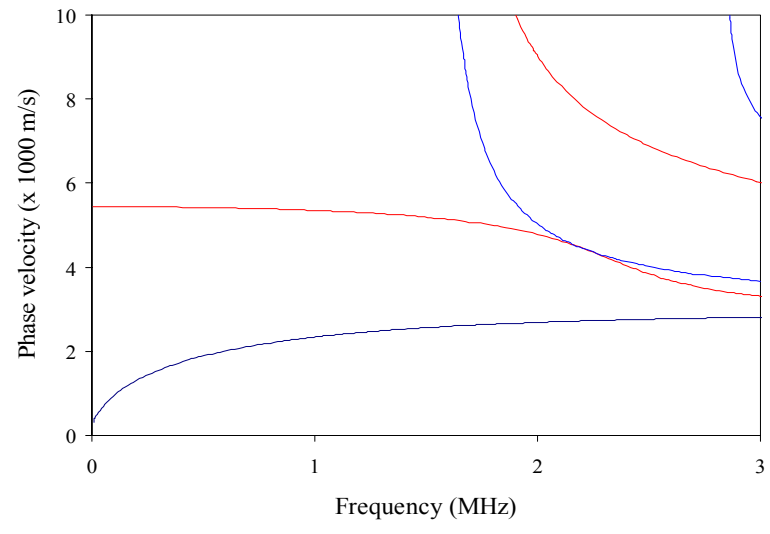

Fig. 4. Phase velocity dispersion curves for an Aluminum cylinder of internal diameter $200 \mathrm{~mm}$ and thickness 1 mm generated using Disperse $e^{25}$

\section{B. Axisymmetric Guided Wave Excitation in Isotropic Cylinders}

In this section, the problem of axisymmetric GW excitation by 3-3 APTs in solid or hollow cylinders (with ringlike cross sections) is considered. Consider an infinitely long isotropic hollow cylinder of outer radius $R_{o}$ and inner radius $R_{i}$ (possibly zero). A 3-3 APT actuator of length $2 a$ is surface-bonded on the outer free surface so that it wraps around the outer circumference. The fibers are oriented along the cylinder axis. The cylindrical co-ordinate system is a natural choice for solving this problem, and the origin is chosen at the center of the mid-plane of the 3-3 APT, as shown in Fig. 5. The starting point of this problem is the governing equations of motion from 3-D elasticity:

$$
(\lambda+\mu) \nabla \nabla \cdot \boldsymbol{u}+\mu \nabla^{2} \boldsymbol{u}+\boldsymbol{f}=\rho \ddot{\boldsymbol{u}}
$$




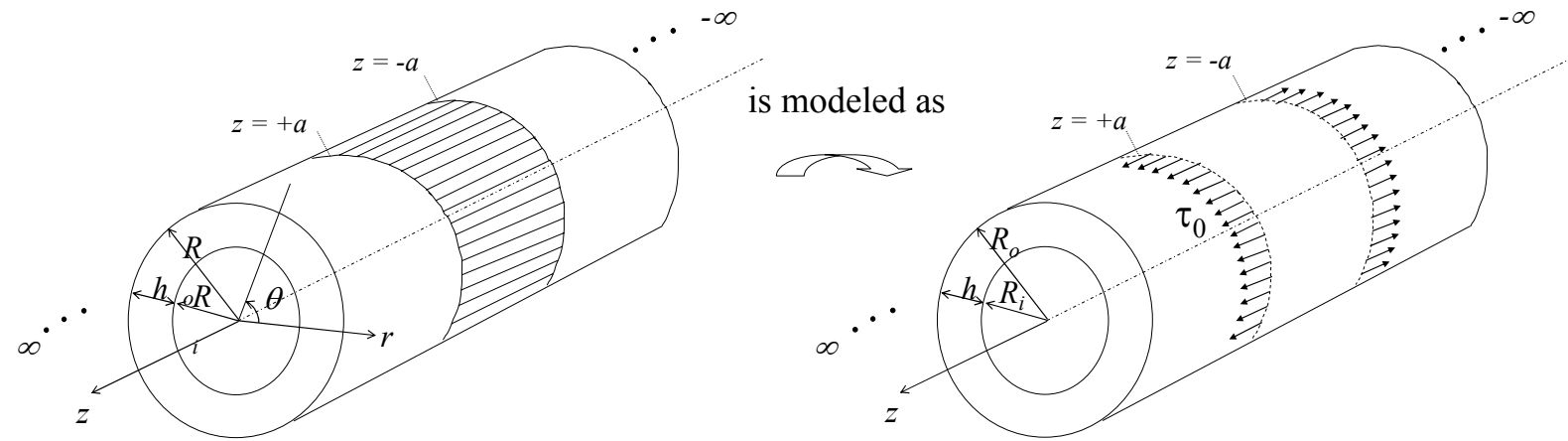

Fig. 5. Configuration of 3-3 APT surface-bonded on a hollow cylinder

In this case, the body force $\boldsymbol{f}=0$. Furthermore, the equations of motion can be decomposed into the Helmholtz components using

$$
\begin{gathered}
\boldsymbol{u}=\nabla \phi+\nabla \times \mathrm{H} \\
\nabla \cdot \mathrm{H}=0
\end{gathered}
$$

It can be shown using Eq. (6), that Eq. (5) is equivalent to the equations:

$$
\begin{array}{rlrl}
\nabla^{2} \phi & =\frac{\ddot{\phi}}{c_{p}^{2}} & ; & c_{p}^{2}=\frac{\lambda+2 \mu}{\rho} \\
\nabla^{2} \mathrm{H}=\frac{\ddot{\mathrm{H}}}{c_{s}^{2}} & ; & c_{s}^{2}=\frac{\mu}{\rho}
\end{array}
$$

In this problem, the conditions $\partial / \partial \theta=0$ and $u_{\theta}=0$ hold. The non-zero displacement components are then given by:

$$
u_{r}=\frac{\partial \phi}{\partial r}-\frac{\partial \mathrm{H}_{\theta}}{\partial z} \quad ; \quad u_{z}=\frac{\partial \phi}{\partial z}+\frac{1}{r} \frac{\partial\left(\mathrm{H}_{\theta} r\right)}{\partial r}
$$

Thus, only the Helmholtz components $\phi$ and $\mathrm{H}_{\theta}$ are required. As before, the response to harmonic excitation at angular frequency $\omega$ is considered. Thus, one obtains:

$$
\frac{\partial^{2}()}{\partial t^{2}}=-\omega^{2}()
$$

Applying the spatial Fourier transform along the $z$-direction yields the following equations:

$$
\frac{\partial^{2} \tilde{\phi}}{\partial r^{2}}+\frac{1}{r} \frac{\partial \tilde{\phi}}{\partial r}+\alpha^{2} \tilde{\phi}=0 \quad ; \quad \frac{\partial^{2} \tilde{\mathrm{H}}_{\theta}}{\partial r^{2}}+\frac{1}{r} \frac{\partial \tilde{\mathrm{H}}_{\theta}}{\partial r}+\left(\beta^{2}-\frac{1}{r^{2}}\right) \tilde{\mathrm{H}}_{\theta}=0
$$

where $\alpha^{2}=\frac{\omega^{2}}{c_{1}^{2}}-\xi^{2}$ and $\beta^{2}=\frac{\omega^{2}}{c_{2}^{2}}-\xi^{2}, \xi$ is the wavenumber along the $z$-direction. The $\sim$ over bar indicates the $z$ direction Fourier transform of the corresponding variable. The solutions to Eqs. (11) are:

$$
\tilde{\phi}=\left(D_{1} J_{0}(\alpha r)+D_{2} Y_{0}(\alpha r)\right) e^{i \omega t} \quad ; \quad \tilde{\mathrm{H}}_{\theta}=\left(D_{3} J_{1}(\beta r)+D_{4} Y_{1}(\beta r)\right) e^{i \omega t}
$$

where $J_{0}$ and $J_{1}$ are the Bessel functions of the first kind and of order 0 and 1 respectively, $Y_{0}$ and $Y_{1}$ are the Bessel functions of the second kind and of order 0 and 1 respectively, $D_{1}, D_{2}, D_{3}$ and $D_{4}$ are constants to be 
determined, using the surface conditions at $r=c_{i}$ and $r=c_{o}$. If the problem under consideration were that of a solid cylinder, i.e., $c_{i}=0$, then $D_{2}, D_{4}=0$ and the other two constants could be found using the surface conditions at $r=c_{o}$. The stresses are related to the displacements through the equations:

$$
\sigma_{r r}=(\lambda+2 \mu) \frac{\partial u_{r}}{\partial r}+\frac{\lambda}{r} u_{r}+\lambda \frac{\partial u_{z}}{\partial z} \quad ; \quad \sigma_{r z}=\mu\left(\frac{\partial u_{r}}{\partial z}+\frac{\partial u_{z}}{\partial r}\right)
$$

Using, Eqs. (11)- (13) gives the stresses in terms of $D_{1}-D_{4}$. The externally applied stresses are:

$$
\begin{array}{lll}
\sigma_{r r}\left(r=c_{o}\right)=\tau_{0} e^{i \omega t}(\delta(z-a)-\delta(z+a)) & ; & \sigma_{r z}\left(r=c_{o}\right)=0 \\
\sigma_{r r}\left(r=c_{i}\right)=0 & ; & \sigma_{r z}\left(r=c_{i}\right)=0
\end{array}
$$

Equating the Fourier transforms of the externally applied stresses to the expressions in terms of $D_{1}-D_{4}$ yields:

$$
\left[\begin{array}{llll}
M_{11} & M_{12} & M_{13} & M_{14} \\
M_{21} & M_{22} & M_{23} & M_{24} \\
M_{31} & M_{32} & M_{33} & M_{34} \\
M_{41} & M_{42} & M_{43} & M_{44}
\end{array}\right]\left[\begin{array}{c}
D_{1} \\
D_{2} \\
D_{3} \\
D_{4}
\end{array}\right]=2 i \tau_{0} \sin \xi a\left[\begin{array}{l}
0 \\
1 \\
0 \\
0
\end{array}\right]
$$

where

$$
\begin{array}{lll}
M_{11}=R_{o}\left(\beta^{2}-\xi^{2}\right) J_{0}\left(\alpha R_{o}\right)-2 \alpha J_{1}\left(\alpha R_{o}\right) & ; & M_{12}=R_{o}\left(\beta^{2}-\xi^{2}\right) Y_{0}\left(\alpha R_{o}\right)-2 \alpha Y_{1}\left(\alpha R_{o}\right) \\
M_{13}=-R_{o} \beta J_{0}\left(\beta R_{o}\right)+J_{1}\left(\beta R_{o}\right) & ; & M_{14}=-R_{o} \beta Y_{0}\left(\beta R_{o}\right)+Y_{1}\left(\beta R_{o}\right) \\
M_{21}=2 i \alpha \xi J_{1}\left(\alpha R_{o}\right) & ; & M_{22}=2 i \alpha \xi Y_{1}\left(\alpha R_{o}\right) \\
M_{23}=\left(\xi^{2}-\beta^{2}\right) J_{1}\left(\beta R_{o}\right) & ; & M_{24}=\left(\xi^{2}-\beta^{2}\right) Y_{1}\left(\beta R_{o}\right)
\end{array}
$$

and the constants $M_{3 i}$ and $M_{4 i}(i=1$ to 4$)$ are obtained by replacing $R_{o}$ by $R_{i}$ in $M_{1 i}$ and $M_{2 i}$ respectively. The constants $D_{i}(i=1$ to 4 ) can be solved for using Cramer's rule, yielding expressions of the form:

$$
D_{i}=\frac{d_{i}(\xi)}{\Delta(\xi)}
$$

where

$$
d_{1}(\xi)=2 i \sin \xi a \cdot \operatorname{det}\left[\begin{array}{llll}
0 & M_{12} & M_{13} & M_{14} \\
1 & M_{22} & M_{23} & M_{24} \\
0 & M_{32} & M_{33} & M_{34} \\
0 & M_{42} & M_{43} & M_{44}
\end{array}\right] \text {, etc. and } \Delta(\xi)=\operatorname{det}\left[\begin{array}{llll}
M_{11} & M_{12} & M_{13} & M_{14} \\
M_{21} & M_{22} & M_{23} & M_{24} \\
M_{31} & M_{32} & M_{33} & M_{34} \\
M_{41} & M_{42} & M_{43} & M_{44}
\end{array}\right]
$$

The final expressions for radial and axial displacements are of the form:

$$
\begin{aligned}
& u_{r}=\int_{-\infty}^{\infty} \frac{1}{\Delta(\xi)}\left[-\alpha d_{1}(\xi) J_{1}(\alpha r)-\alpha d_{2}(\xi) Y_{1}(\alpha r)+i \xi d_{3}(\xi) J_{1}(\beta r)+i \xi d_{4}(\xi) Y_{1}(\beta r)\right] e^{-i(\xi z-\omega t)} d \xi \\
& u_{z}=\int_{-\infty}^{\infty} \frac{1}{\Delta(\xi)}\left[-i \xi d_{1}(\xi) J_{0}(\alpha r)-i \xi d_{2}(\xi) Y_{0}(\alpha r)+\beta d_{3}(\xi) J_{0}(\beta r)+\beta d_{4}(\xi) Y_{0}(\beta r)\right] e^{-i(\xi z-\omega t)} d \xi
\end{aligned}
$$




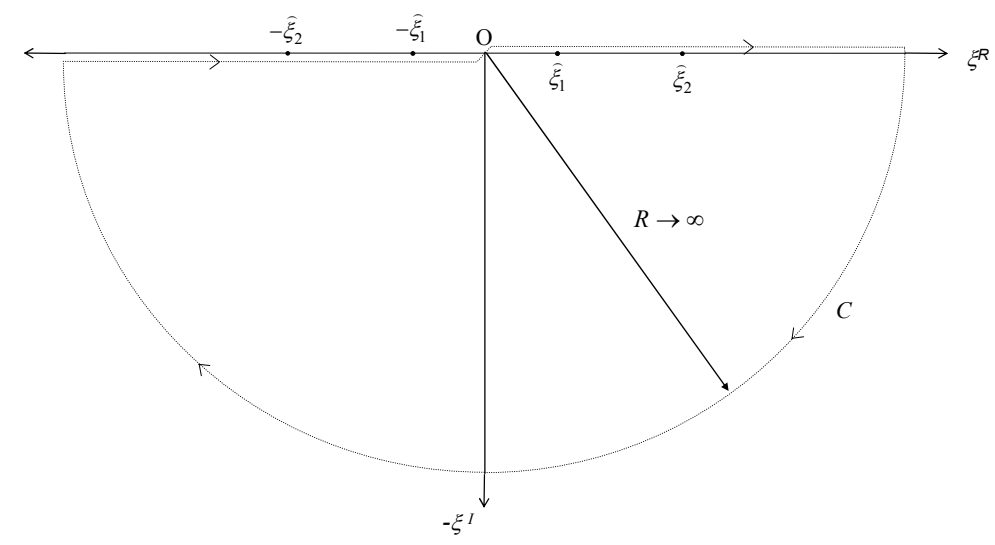

Fig. 6. Contour integral in the complex $\xi$-plane to invert the displacement integrals using residue theory

The integral along the real $\xi$-axis is replaced by a contour integral in the complex $\xi$-plane. The values of $z$ will determine the shape of the contour. For example, if $z>a$ then contributions from negative wavenumbers are not allowed on physical grounds, hence the integral must only include the residues at positive wavenumbers, as shown in Fig. 6. The integrands in Eq. (19) are singular at the roots of the dispersion equation $\Delta(\xi)=0$, designated $\hat{\xi}$. These can be obtained from the dispersion curves for the cylinder under consideration, as shown in Fig. 4. Using the residue theorem for the first integral in Eq. (19) yields in this case:

$$
\int_{-\infty}^{\infty} I d \xi+\int_{C} I d \xi=-\pi i \sum_{\xi} \operatorname{Res}(\mathrm{I}(\bar{\xi}))
$$

where

$$
I=\frac{1}{\Delta(\xi)}\left[-\alpha d_{1}(\xi) J_{1}(\alpha r)-\alpha d_{2}(\xi) Y_{1}(\alpha r)+i \xi d_{3}(\xi) J_{1}(\beta r)+i \xi d_{4}(\xi) Y_{1}(\beta r)\right] e^{-i(\xi z-\omega t)}
$$

$C$ is the semi-circular contour in the lower half-plane while "Res" stands for the residue of the integrand at a singularity of $I$. The contribution from $C$ vanishes as the radius of the surface $R \rightarrow \infty$, as explained in Miklowitz ${ }^{27}$ for a similar plane-wave excitation problem. Thus, the following expressions are obtained for displacement in the region $z>a$ :

$$
\begin{aligned}
& u_{r}=\sum_{\hat{\xi}} \frac{-\pi i e^{-i(\hat{\xi} z-\omega t)}}{\Delta^{\prime}(\hat{\xi})}\left[-\alpha d_{1}(\hat{\xi}) J_{1}(\alpha r)-\alpha d_{2}(\hat{\xi}) Y_{1}(\alpha r)+i \hat{\xi} d_{3}(\hat{\xi}) J_{1}(\beta r)+i \hat{\xi} d_{4}(\hat{\xi}) Y_{1}(\beta r)\right] \\
& u_{z}=\sum_{\hat{\xi}} \frac{-\pi i e^{-i(\hat{\xi} z-\omega t)}}{\Delta^{\prime}(\hat{\xi})}\left[-i \hat{\xi} d_{1}(\hat{\xi}) J_{0}(\alpha r)-i \hat{\xi} d_{2}(\hat{\xi}) Y_{0}(\alpha r)+\beta d_{3}(\hat{\xi}) J_{0}(\beta r)+\beta d_{4}(\hat{\xi}) Y_{0}(\beta r)\right]
\end{aligned}
$$

\section{3-D Guided Wave Excitation in Isotropic Plates}

The final configuration that will be examined is that of 3-3 APTs surface-bonded on isotropic plates. Consider a rectangular 3-3 APT of dimensions $2 a_{1}$ and $2 a_{2}$ surface-bonded on the surface of an infinite isotropic plate of thickness $2 b$, as shown in Fig. 7. The coordinate axis system is chosen such that the $x_{3}$-axis is normal to the plane of the plate and the $x_{2}$-axis is along the fiber direction. The starting point is the equations of motion in terms of the Helmholtz components, i.e., Eqs. (7) and (8). The double spatial Fourier transform is used to ease solution of this problem. For a generic function of two spatial coordinates $\varphi$, it is defined by:

$$
\bar{\varphi}\left(\xi_{1}, \xi_{2}\right)=\int_{-\infty}^{\infty} \int_{-\infty}^{\infty} \varphi\left(x_{1}, x_{2}\right) e^{i\left(\xi_{1} x_{1}+\xi_{2} x_{2}\right)} d x_{1} d x_{2}
$$


and the inverse is given by:

$$
\varphi\left(x_{1}, x_{2}\right)=\frac{1}{4 \pi^{2}} \int_{-\infty}^{\infty} \int_{-\infty}^{\infty} \bar{\varphi}\left(\xi_{1}, \xi_{2}\right) e^{-i\left(\xi_{1} x_{1}+\xi_{2} x_{2}\right)} d \xi_{1} d \xi_{2}
$$

Applying the double spatial Fourier transform on Eqs. (7) and (8) and considering harmonic excitation as before, one obtains the following equations:

$$
\begin{gathered}
\left(-\xi_{1}^{2}-\xi_{2}^{2}\right) \bar{\phi}+\frac{d^{2} \bar{\phi}}{d x_{3}^{2}}=-\frac{\omega^{2}}{c_{p}^{2}} \bar{\phi} \\
\left(-\xi_{1}^{2}-\xi_{2}^{2}\right) \overline{\mathbf{H}}+\frac{d^{2} \overline{\mathbf{H}}}{d x_{3}^{2}}=-\frac{\omega^{2}}{c_{s}^{2}} \overline{\mathbf{H}}
\end{gathered}
$$

Let:

$$
\left(-\xi_{1}^{2}-\xi_{2}^{2}\right)+\frac{\omega^{2}}{c_{p}^{2}}=\alpha^{2} \quad ; \quad\left(-\xi_{1}^{2}-\xi_{2}^{2}\right)+\frac{\omega^{2}}{c_{s}^{2}}=\beta^{2}
$$

The solutions of Eqs. (25) and (26) are of the form (the $e^{i \omega t}$ factor is dropped from all subsequent equations and will be brought back in the final expression):

$$
\begin{array}{rll}
\bar{\phi} & =C_{1} \sin \alpha x_{3}+C_{2} \cos \alpha x_{3} \quad ; \quad & \overline{\mathrm{H}}_{1}=C_{3} \sin \beta x_{3}+C_{4} \cos \beta x_{3} \\
\overline{\mathrm{H}}_{2}=C_{5} \sin \beta x_{3}+C_{6} \cos \beta x_{3} & ; & \overline{\mathrm{H}}_{3}=C_{7} \sin \beta x_{3}+C_{8} \cos \beta x_{3}
\end{array}
$$

Furthermore, it can be shown that the constants $C_{2}, C_{3}, C_{5}$ and $C_{8}$ are associated with symmetric modes and that the constants $C_{1}, C_{4}, C_{6}$ and $C_{7}$ are associated with antisymmetric modes. For the subsequent analysis, only the symmetric modes are considered. The contributions from antisymmetric modes can be derived analogously. The linear strain-displacement relation and the constitutive equations for linear elasticity yield:

$$
\varepsilon_{i j}=\frac{1}{2}\left(u_{i, j}+u_{j, i}\right) \quad ; \quad \sigma_{i j}=\lambda \varepsilon_{k k} \delta_{i j}+2 \mu \varepsilon_{i j}
$$

Using Eqs.(6), (28) and (29), it can be shown that the transformed stresses at $x_{3}=b$ are:

$$
\begin{aligned}
& \bar{\sigma}_{33}=\mu\left[C_{2}\left(\xi_{1}^{2}+\xi_{2}^{2}-\beta^{2}\right) \cos \alpha b+C_{3}\left(2 i \mu \xi_{2} \beta\right) \cos \beta b+C_{5}\left(-2 i \xi_{1} \beta\right) \cos \beta b\right] \\
& \bar{\sigma}_{32}=\mu\left[C_{2}\left(2 i \xi_{2} \alpha\right) \sin \alpha b+C_{3}\left(\xi_{2}^{2}-\beta^{2}\right) \sin \beta b+C_{5}\left(-\xi_{1} \xi_{2}\right) \sin \beta b+C_{8}\left(-i \xi_{1} \beta\right) \sin \beta b\right] \\
& \bar{\sigma}_{31}=\mu\left[C_{2}\left(2 i \alpha \xi_{1}\right) \sin \alpha b+C_{3}\left(\xi_{1} \xi_{2}\right) \sin \beta b+C_{5}\left(\beta^{2}-\xi_{1}^{2}\right) \sin \beta b+C_{8}\left(i \xi_{2} \beta\right) \sin \beta b\right]
\end{aligned}
$$

Since the 3-3 APT is modeled as causing shear traction along the fiber direction, the surface conditions are:

$$
\begin{aligned}
& \sigma_{31}=0 \\
& \sigma_{32}=\tau_{0} e^{i \omega t}\left[H e\left(x_{1}+a_{1}\right)-H e\left(x_{1}-a_{1}\right)\right]\left[\delta\left(x_{2}-a_{2}\right)-\delta\left(x_{2}+a_{2}\right)\right] \\
& \sigma_{33}=0
\end{aligned}
$$




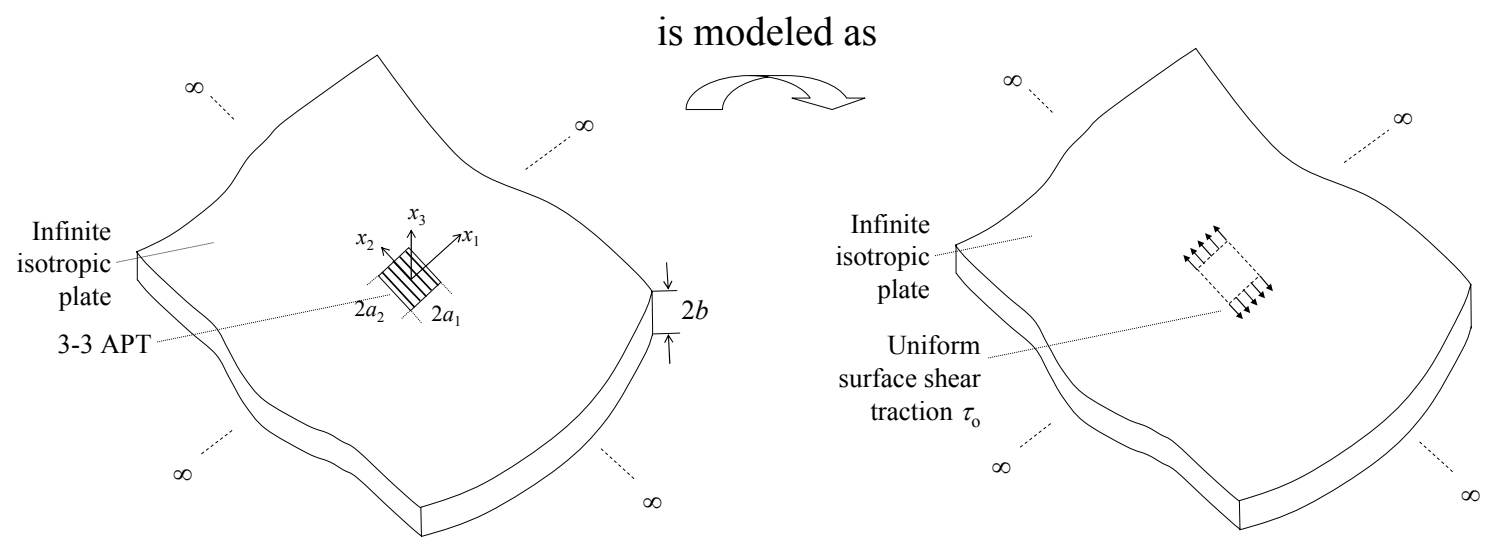

Fig. 7. Model for 3-3 APT to solve for the excited GW field in isotropic plates

Equating Eqs. (30) and (31) would give three equations in four unknowns. The fourth equation results from the divergence condition on $\mathrm{H}$, and consequently, $\overline{\mathrm{H}}$, given by:

$$
\frac{\partial \overline{\mathrm{H}}_{1}}{\partial x_{1}}+\frac{\partial \overline{\mathrm{H}}_{2}}{\partial x_{2}}+\frac{\partial \overline{\mathrm{H}}_{3}}{\partial x_{3}}=0
$$

Using Eqs. (28) in (32) and evaluating at $x_{3}=b$ gives:

$$
C_{3}\left(-i \xi_{1} \sin \beta b\right)+C_{5}\left(-i \xi_{2} \sin \beta b\right)+C_{8}(-\beta \sin \beta b)=0
$$

With four equations and four unknowns, the unknown constants $C_{2}, C_{3}, C_{5}$ and $C_{8}$ can be solved for from the matrix equation:

$$
\left[\begin{array}{cccc}
\left(\xi_{1}^{2}+\xi_{2}^{2}-\beta^{2}\right) \cos \alpha b & 2 i \xi_{2} \beta \cos \beta b & -2 i \xi_{1} \beta \cos \beta b & 0 \\
2 i \xi_{2} \alpha \sin \alpha b & \left(\xi_{2}^{2}-\beta^{2}\right) \sin \beta b & -\xi_{1} \xi_{2} \sin \beta b & -i \xi_{1} \beta \sin \beta b \\
2 i \xi_{1} \alpha \sin \alpha b & \xi_{1} \xi_{2} \sin \beta b & \left(\beta^{2}-\xi_{1}^{2}\right) \sin \beta b & i \xi_{2} \beta \sin \beta b \\
0 & -i \xi_{1} \sin \beta b & -i \xi_{2} \sin \beta b & -\beta \sin \beta b
\end{array}\right]\left[\begin{array}{c}
C_{2} \\
C_{3} \\
C_{5} \\
C_{8}
\end{array}\right]=\frac{1}{\mu}\left[\begin{array}{c}
0 \\
\bar{F}_{2}\left(\xi_{1}, \xi_{2}\right) \\
\bar{F}_{1}\left(\xi_{1}, \xi_{2}\right) \\
0
\end{array}\right]
$$

Solving for the constants and applying the inverse Fourier transform ultimately yields the following expressions for the transformed displacement components on the free surface $x_{3}=b$ :

$$
\begin{aligned}
& u_{1}^{S}\left(x_{3}=b\right)=\int_{0}^{\infty} \int_{0}^{2 \pi} \frac{4 \tau_{0}}{\pi^{2} i \mu} \frac{M_{S}\left(\xi^{S}\right)}{\left(\beta \sin \beta b \cdot D_{S}\left(\xi^{S}\right)\right)} \sin \gamma \sin \left(\xi^{S} \cos \gamma a_{1}\right) \sin \left(\xi^{S} \sin \gamma a_{2}\right) e^{-i \xi^{S}\left(\cos \gamma x_{1}+\sin \gamma x_{2}-\omega t\right)} d \gamma d \xi \\
& u_{2}^{S}\left(x_{3}=b\right)=\int_{0}^{\infty} \int_{0}^{\infty 2 \pi} \frac{-4 \tau_{0}}{\pi^{2} i \mu} \frac{\cos \beta b}{\left(\beta \sin \beta b \cdot D_{S}\left(\xi^{S}\right)\right)} \frac{\sin \left(\xi^{S} \cos \gamma a_{1}\right) \sin \left(\xi^{S} \sin \gamma a_{2}\right) L_{S}\left(\xi^{S}, \gamma\right)}{\cos \gamma} e^{-i \xi^{S}\left(\cos \gamma x_{1}+\sin \gamma x_{2}-\omega t\right)} d \gamma d \xi \\
& u_{3}^{S}\left(x_{3}=b\right)=\int_{0}^{\infty} \int_{0}^{2 \pi} \frac{-4 \tau_{0}}{\pi^{2} \mu} \frac{T_{S}\left(\xi^{S}\right)}{\xi D_{S}\left(\xi^{S}\right)} \tan \gamma \sin \left(\xi^{S} \cos \gamma a_{1}\right) \sin \left(\xi^{S} \sin \gamma a_{2}\right) e^{-i \xi^{S}\left(\cos \gamma x_{1}+\sin \gamma x_{2}-\omega t\right)} d \gamma d \xi
\end{aligned}
$$




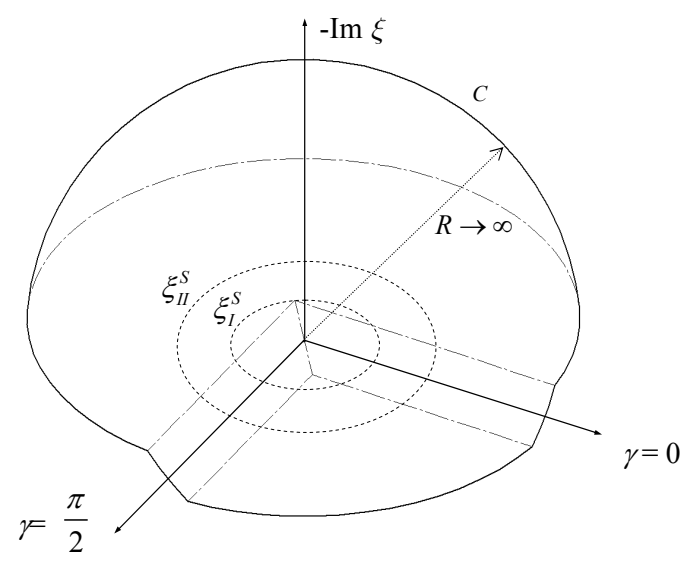

Fig. 8. Surface construction in the complex $\xi-\gamma$ space

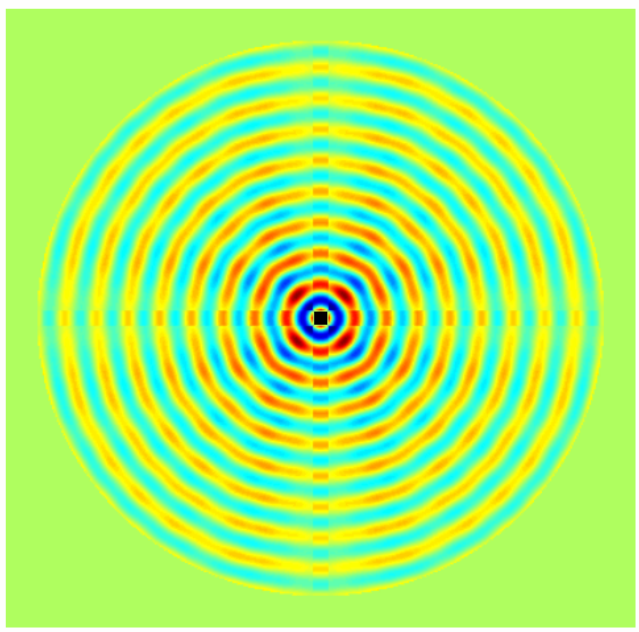

Fig. 10. Harmonic wave field $\left(u_{3}\right)$ by $0.5 \mathrm{~cm} \times 0.5$ cm square uniformly poled piezo-actuator

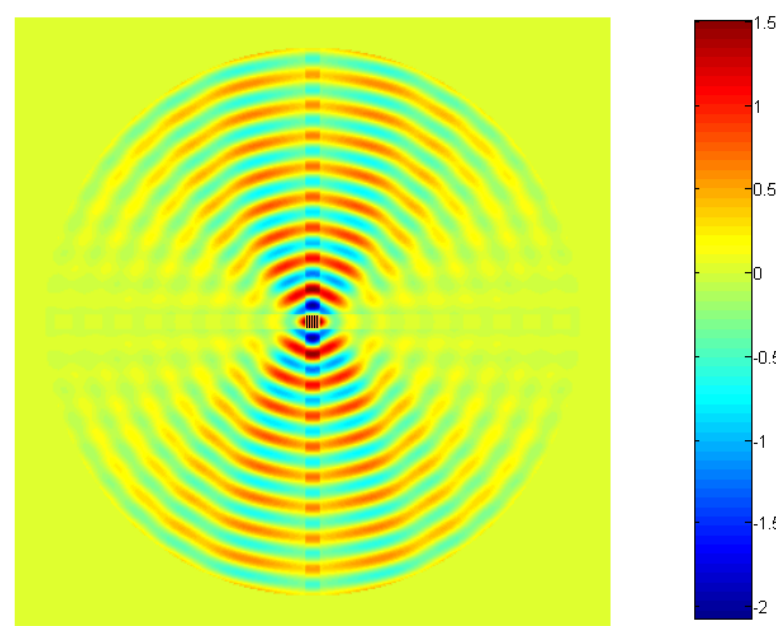

Fig. 9. Harmonic wave field $\left(u_{3}\right)$ by square 3-3 APT at $100 \mathrm{kHz}, A_{0}$ mode (the plate area shown is $10 \mathrm{~cm} \times 10 \mathrm{~cm}$, and the actuators are $0.5 \mathrm{~cm} \times 0.5$ $\mathrm{cm})$. The fibers are along the vertical direction.
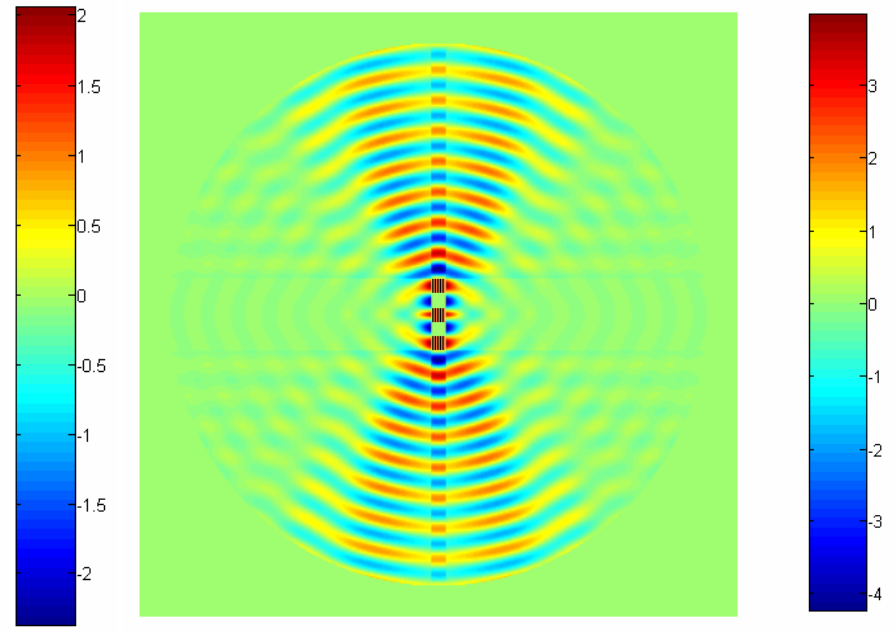

Fig. 11. Harmonic wave field $\left(u_{3}\right)$ by threeelement comb array of $0.5 \mathrm{~cm} \times 0.5 \mathrm{~cm}$ square $3-3$

APTs

In this case, to obtain the 2-D spatial inverse Fourier transform, residue calculus is used again as in the previous section. However, in this case, the integral is two-dimensional and the integral in the real $\xi-\gamma$ plane is replaced by a surface integral in the complex $\xi-\gamma$ space. The sign of $x_{1}$ and $x_{2}$ will determine the shape of the contour. For example, if $x_{1}>a_{1}, x_{2}>a_{2}$ then contributions from negative wavenumbers are not allowed on physical grounds, hence the surface must only include the first quadrant, i.e., $\gamma \in(0, \pi / 2)$ and the lower half of the complex $\xi-\gamma$ space as shown in Fig. 8. Applying the residue theorem as in the previous section, the following expressions are obtained for the displacement components:

$$
\begin{aligned}
& u_{1}^{S}\left(x_{3}=b\right)=\sum_{\xi^{S}} \frac{-4 \tau_{0}}{\pi \mu} \frac{M_{S}\left(\xi^{S}\right)}{\left(\beta \sin \beta b \cdot D_{S}\left(\xi^{S}\right)\right)^{\prime}} e^{i \omega t} \int_{0}^{\frac{\pi}{2}} \sin \gamma \sin \left(\xi^{S} \cos \gamma a_{1}\right) \sin \left(\xi^{S} \sin \gamma a_{2}\right) e^{-i \xi^{S}\left(\cos \gamma x_{1}+\sin \gamma x_{2}\right)} d \gamma \\
& u_{2}^{S}\left(x_{3}=b\right)=\sum_{\xi^{S}} \frac{4 \tau_{0}}{\pi \mu} \frac{\cos \beta b}{\left(\beta \sin \beta b \cdot D_{s}\left(\xi^{S}\right)\right)^{\prime}} e^{i \omega t} \int_{0}^{\frac{\pi}{2}} \frac{\sin \left(\xi^{S} \cos \gamma a_{1}\right) \sin \left(\xi^{S} \sin \gamma a_{2}\right) L_{S}\left(\xi^{s}, \gamma\right)}{\cos \gamma} e^{-i \xi^{S}\left(\cos \gamma x_{1}+\sin \gamma x_{2}\right)} d \gamma
\end{aligned}
$$




$$
u_{3}^{S}\left(x_{3}=b\right)=\sum_{\xi^{S}} \frac{4 i \tau_{0}}{\pi \mu} \frac{T_{S}\left(\xi^{S}\right)}{\xi D_{S}^{\prime}\left(\xi^{S}\right)} e^{i \omega t} \int_{0}^{\frac{\pi}{2}} \tan \gamma \sin \left(\xi^{S} \cos \gamma a_{1}\right) \sin \left(\xi^{S} \sin \gamma a_{2}\right) e^{-i \xi^{S}\left(\cos \gamma x_{1}+\sin \gamma x_{2}\right)} d \gamma
$$

where

$$
\begin{aligned}
& M_{S}(\xi)=\xi^{2} \cos \beta b\left[\left(\xi^{2}-3 \beta^{2}\right) \cos \alpha b \sin \beta b+4 \alpha \beta \sin \alpha b \cos \beta b\right] \\
& L_{S}(\xi, \gamma)=\left[\left(\xi^{2} \cos ^{2} \gamma-\beta^{2}\right)^{2}+\xi^{2} \sin ^{2} \gamma\left(\xi^{2} \cos ^{2} \gamma+\beta^{2}\right)\right] \cos \alpha b \sin \beta b+4 \alpha \beta \xi^{2} \cos ^{2} \gamma \sin \alpha b \cos \beta b \\
& T_{S}(\xi)=\xi^{2}\left(\beta^{2}-\xi^{2}\right) \cos \alpha b \sin \beta b-2 \alpha \beta \xi^{2} \cos \beta b \sin \alpha b
\end{aligned}
$$

Similarly, the contributions from the antisymmetric modes can be found. It is interesting to note that the expressions for $u_{1}$ and $u_{2}$ have contributions from the roots of both the Rayleigh-Lamb equation $\left(D_{S}=0\right)$ as well as the equation for horizontally polarized or SH-waves ( $\sin \beta b=0$ ), whereas that for $u_{3}$ has contributions only from the roots of the Rayleigh-Lamb equation. This is logical in hindsight, since SH-waves do not cause out-of-plane displacements. An approximate closed form solution can be obtained for the far field using the method of stationary phase. As explained in $\mathrm{Graff}^{28}$, for large $r$ :

$$
\int_{\psi_{1}}^{\psi_{2}} f(\psi) e^{i r h(\psi)} d \psi \approx \sqrt{\frac{2 \pi}{r h^{\prime \prime}\left(\psi_{0}\right)}} f\left(\psi_{0}\right) e^{i\left(r \psi_{0}+\pi / 4\right)}
$$

where $h^{\prime}\left(\psi_{0}\right)=0, f()$ is an arbitrary function, and $\psi_{1}$ and $\psi_{2}$ are arbitrary end-points of the interval of integration, which contains $\psi_{0}$. Hence, the following asymptotic expression holds for particle displacement along the 1-direction in the far field in the region $x_{1}>a_{1}, x_{2}>a_{2}$ :

$$
u_{1}^{S}\left(x_{3}=b\right)=\sum_{\xi^{S}} \frac{-4 \tau_{0}}{\pi \mu} \cdot \sqrt{\frac{2 \pi}{\xi^{S} r}} \cdot \frac{M_{S}\left(\xi^{S}\right)}{\left(\beta \sin \beta b \cdot D_{S}\left(\xi^{S}\right)\right)^{\prime}} \sin \theta \cdot \sin \left(\xi^{S} \cos \theta a_{1}\right) \cdot \sin \left(\xi^{S} \sin \theta a_{2}\right) e^{-i\left(\xi^{S} r+\pi / 4^{-\omega t)}\right.}
$$

where $\theta=\tan ^{-1}\left(x_{2} / x_{1}\right)$ and $r=\sqrt{x_{1}^{2}+x_{2}^{2}}$. This indicates that the GW field tends to a circular crested field with angularly dependent amplitude in the far field.

In other regions of the plate, the included region of the contour will change. For example in the region, $x_{1}>a_{1},-a_{2}<x_{2}<a_{2}$, due to the presence of both positive and negative wavenumbers along the $x_{2}$-direction and only positive wavenumbers along the $x_{1}$-direction, the included region of the contour will be from $\gamma=-\pi / 2$ to $\gamma=\pi / 2$.

The predicted harmonic out-of-plane displacement patterns due to excitation of the $\mathrm{A}_{0}$ Lamb mode at $100 \mathrm{kHz}$ in a 2-mm Aluminum plate by a square 3-3 APT and a square uniformly poled piezo-actuator are shown in Fig. 9 and Fig. 10, respectively. For ease of visualization, only $10 \mathrm{~cm} \times 10 \mathrm{~cm}$ of the plate is shown and the field was set to zero for radius $r>9 \mathrm{~cm}$ in both cases. These illustrate how the GW field tends to a circular crested wave field with directionally dependent amplitude at large distances from the actuator. In addition, they highlight the directionally focused nature of the GW field from 3-3 APTs. The waves propagate in a roughly collimated beam in a limited sector centered about the fiber direction. This directionality is expected to be refined even more if the electrode pattern of the transducer is designed in a comb-transducer like fashion (see Fig. 11). This can be achieved by designing the clusters of electrode fingers spaced at intervals equal to half of the wavelength of the excited GW. Such a comb transducer also has much better modal selectivity being more tuned to excite a particular wavelength chosen by design.

\section{3-3 Anisotropic Piezocomposite Transducers as Sensors}

In this case, the underlying assumption is that the 3-3 APT senses the average in-plane extensional strain along the fiber direction over its length, and is insensitive to the other strain components. Let the direction along which its fibers are oriented be the 2-direction of the structure. By the IEEE standard on piezoelectricity ${ }^{29}$, this being the 
poling direction, it is the 3-direction of the 3-3 APT. Consider the charge accumulated by a 3-3 APT sensor surfacebonded on a structure in a GW field. This is given by:

$$
Q_{c}=\frac{E_{33_{c}} k_{c} \varepsilon_{0} g_{33}}{1-v_{c}} \int_{S_{c}} \varepsilon_{22} d S
$$

where $E_{33_{c}}$ is the in-plane Young's modulus of the sensor material, $k_{c}$ is the dielectric constant of the sensor material, $v_{c}$ is the Poisson's ratio of the sensor material, $\varepsilon_{0}$ is the permittivity of vacuum, $g_{33}$ is a piezoelectric constant $^{29}, \varepsilon_{22}$ is the in-plane extensional surface strain along the structure's 2-direction and $S_{c}$ is the surface area of the sensor. An important assumption made here is that the sensor is infinitely compliant and does not disturb the GW field. This is reasonably satisfied in the case of 3-3 APTs if the product of the sensor's thickness and Young's modulus is small compared to that of the plate on which it is surface-bonded and if it is of small size. If the capacitance of the sensor is given by $C_{c}$, the output voltage response of the piezo-sensor is obtained as:

$$
V_{c}=\frac{Q_{c}}{C_{c}}=\frac{E_{33_{c}} k_{c} \varepsilon_{0} g_{33}}{C_{c} \cdot\left(1-v_{c}\right)} \int_{S_{c}} \varepsilon_{22} d S
$$

\section{Experimental Tests and Results}

To examine the validity of these derived theoretical models, experiments were conducted with Aluminum beam and plate specimens. In these experiments, MFCs were bonded on either surface at the center and used as actuators. For both specimens, experiments were conducted to examine the correlation between theoretical and experimental frequency response functions. The first transmitted pulse sensed by a surface-bonded MFC sensor at some distance from the center was monitored. Two sets of experiments were conducted. In the first set, the actuators were excited in phase to excite symmetric modes while in the second they were excited out of phase in order to excite the antisymmetric modes. These actuators were powered with a 3.5-cycle Hann-windowed sinusoidal toneburst over a range of center frequencies. The highest excitation frequency was well below the cut-off frequency of the first symmetric Lamb mode in the first set. In the second set, it was well below the cut-off frequency of the first antisymmetric Lamb mode and the first antisymmetric SH-mode. Thus, the $\mathrm{S}_{0}$ mode was predominantly excited in the first set while the $A_{0}$ mode was predominantly excited in the second set. Apart from these experiments, an experiment was also performed using a laser vibrometer to test the accuracy of the theoretically predicted spatial variation of the GW field excited by MFCs in plate structures. The values of the Aluminum material properties used for the theoretical curves were as follows: Young's modulus $E_{A l}=70 \mathrm{GPa}$, Poisson's ratio $v=0.33$, density $\rho=$ $2700 \mathrm{~kg} / \mathrm{m}^{3}$.

\section{A. Beam Experiment for Frequency Response Function}

A 1-mm thick Aluminum alloy strip clamped at both ends was instrumented with three MFCs, each $0.2 \mathrm{~mm}$ thick, as illustrated in Fig. 12. The actuators were excited with a 5-V (peak-to-peak) signal and the average amplitude of the sensor response over 16 samples was noted to reduce the noise levels. To predict the theoretical sensor response trend versus frequency, one needs to use the value $2 a=3.2 \mathrm{~cm}$ (which is the length of the active area of the MFC) in the beam formulation. Only the contributions from the $\mathrm{S}_{0}$ mode were included for the first set. Similarly, only contributions of the $\mathrm{A}_{0}$ mode were considered for the second set. The harmonic sensor response, found using Eq. (44) should also be integrated over the frequency bandwidth of excitation for calculating the response to a 3.5-cycle sinusoidal toneburst signal. The theoretical (also referred to as analytical) and experimental results are compared in Fig. 13. Both curves are normalized to the peak response amplitude over the considered frequency range.

\section{B. Plate Experiment for Frequency Response Function}

For the plate experiment, a 1-mm thick Aluminum plate of dimensions $50 \mathrm{~cm} \times 50 \mathrm{~cm}$ and clamped on all sides was instrumented with three MFCs, each $0.3 \mathrm{~mm}$ thick, as illustrated in Fig. 14. As in the previous experiment, two of these MFCs at the center were used as actuators while the third was used as a sensor. In this case, the actuators were excited with an 18-V (peak-to-peak) signal. For each reading, the excitation signal was repeated at a frequency of $1 \mathrm{~Hz}$ (this was small enough so that there was no interference between successive repetitions) and the averaged 


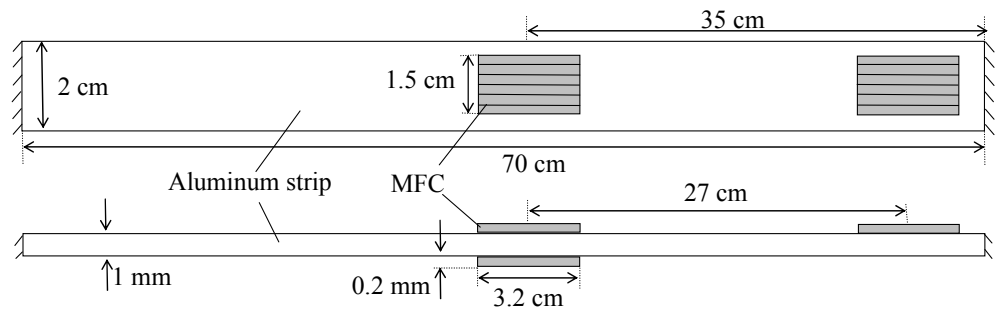

Fig. 12. Illustration of thin Aluminum strip instrumented with MFCs
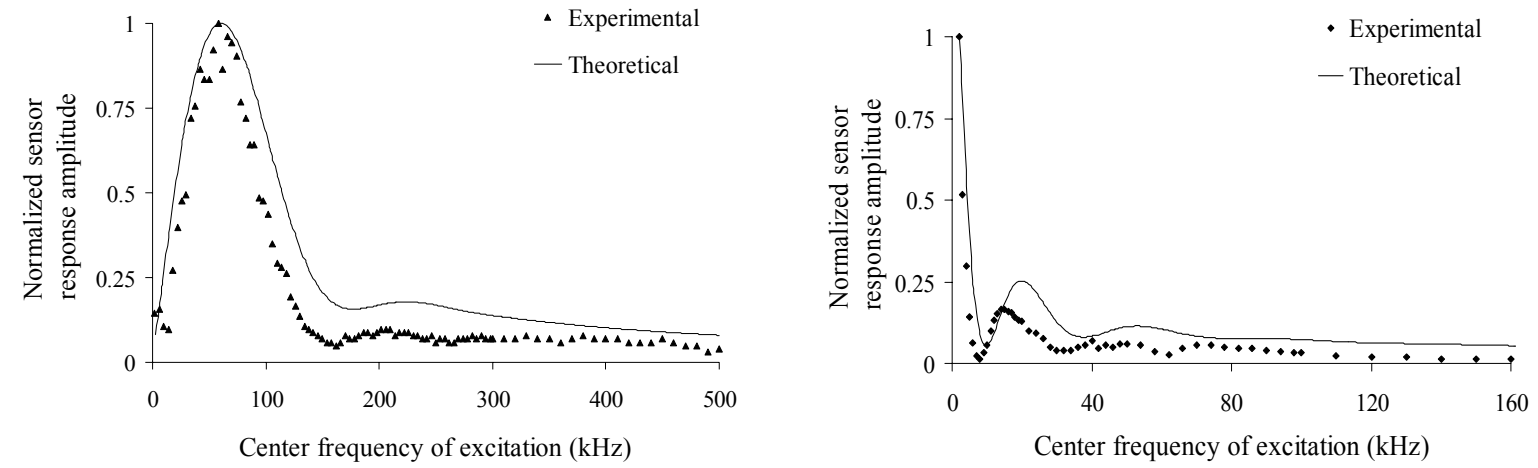

Fig. 13. Theoretical and experimental normalized sensor response over various frequencies in the beam experiment for $S_{0}$ (left) and $A_{0}$ (right) modes

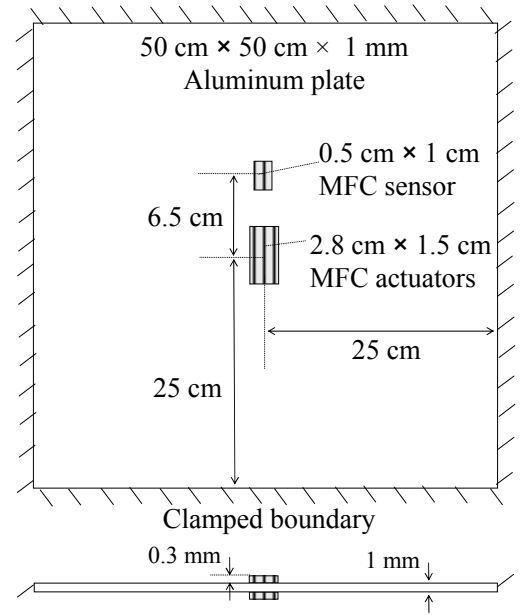

Fig. 14. Schematic of Aluminum plate experiment
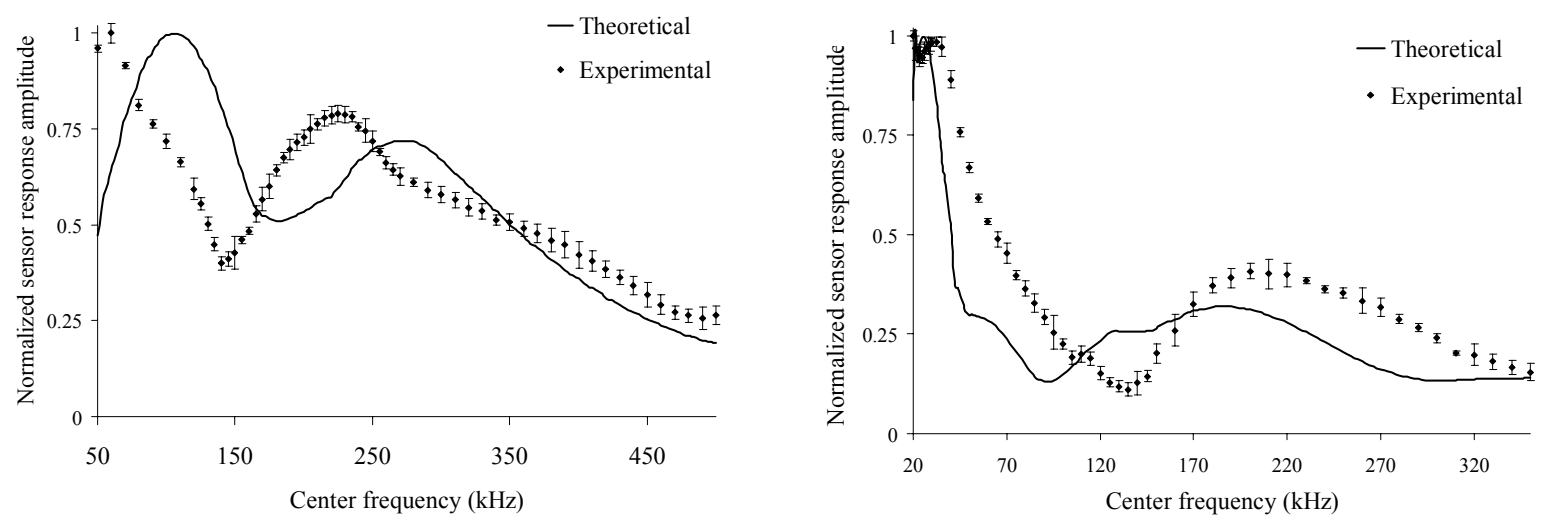

Fig. 15. Theoretical and experimental normalized sensor response over various center frequencies in the plate experiment for $S_{0}$ (left) and $A_{0}$ (right) modes

American Institute of Aeronautics and Astronautics 
signal over 64 samples was used. To predict the theoretical sensor response trend versus frequency, one needs to use the value $2 a_{1}=1.5 \mathrm{~cm}$ and $2 a_{2}=2.8 \mathrm{~cm}$ (which is the size of the active area of the MFC) in the plate formulation. As in the beam experiment, only the contributions from the relevant mode were included for each set. It was found from analysis that the contribution of the $\mathrm{SH}$-modes to the sensor response was negligible and, therefore, these were ignored. This is logical, since the MFC sensors are sensitive to only extensional strain while SH-waves predominantly cause shear strains. The theoretical and experimental results are compared in Fig. 15. Again, both curves are normalized to the peak response amplitude over the frequency range considered. The error bars based on the standard deviation of the amplitudes over the 64 samples (capturing $99.73 \%$ of the data points), and normalized by the peak amplitude are also shown.

\section{Plate Experiment using Laser Vibrometer}

To test the theoretically-predicted focusing capability of the MFC along its fiber direction in plate structures, an experiment was conducted using a Polytec scanning laser vibrometer system that employed a Polytec OFV-303 sensor head and OFV-3001-S controller. The same plate specimen as described in Section III.B was used. Again, the actuators were excited with a 18-V (peak-to-peak) 3.5-cycle Hann windowed sinusoidal toneburst signal. However, for this experiment, the center frequency was kept fixed at $30 \mathrm{kHz}$ and the actuators were excited out-of-phase to excite the $\mathrm{A}_{0}$ mode predominantly. The laser vibrometer measured out-of-plane surface velocity signals at a chosen point, and was equipped with a computer-controlled scanning head (Polytec OFV-040) so that the scan point could be swept with precision over the plate area. Measurements were done over a grid spanning a quarter section of the plate surface (up to $20 \mathrm{~cm}$ from each symmetry axis), since the field is expected to be symmetrical about the two axes in the plane of the plate. The grid spacing was $0.6 \mathrm{~cm}$ along the fiber direction (which is a third of the $\mathrm{A}_{0}$ mode wavelength at $30 \mathrm{kHz}$ ). Along the other direction in the plane of the plate near the actuator, the spacing was $0.5 \mathrm{~cm}$ (for the first four columns of the grid starting from the symmetry axis). Beyond this region, the spacing was $1 \mathrm{~cm}$. In addition, as in the previous section, the excitation signal was repeated 64 times for each point at a frequency of $1 \mathrm{~Hz}$ and the averaged signal was recorded. Furthermore, wavelet-denoising using the discrete Meyer wavelet was employed to cleanse the signals. The experimentally obtained surface plots at three particular time instants over the quarter section of the plate are shown in Fig. 16a (normalized to the peak value of surface velocity over the plate in the time span up to $200 \mu \mathrm{s}$ ). The surface plots for the same GW fields obtained using the theoretical model developed in this work are shown adjacent to these in Fig. 16b. These are also normalized to the theoretically predicted peak velocity over the plate area in the same time span. These plots were generated assuming pure $\mathrm{A}_{0}$ mode excitation. SH-modes were not considered since they do not cause out-of-plane displacements.

\section{Discussion and Sources of Error}

\section{A. Frequency Response Function Experiments}

In the beam experiment, for the symmetric mode, the peak response frequency is well captured by the model. In addition, the qualitative trend of the sensor response with varying frequency is also captured. Similar conclusions hold for the antisymmetric mode. In this case, however, the peak response frequency is the lowest frequency of testing. The qualitative prediction of the trend of the response is good, albeit with some marginal quantitative error in the location and relative magnitude of peaks. The frequency at which the second peak occurs is slightly overestimated for both modes.

In the plate frequency response experiment, there is qualitative agreement in the trend between the theoretical and experimental results. However, there is some error in the prediction of frequencies of peak sensor response. The frequencies of the peaks are over-estimated for the $S_{0}$ mode while they are under-estimated for the $A_{0}$ mode. Evidently, the extent of disagreement between theory and experiment is greater in the plate experiment than in the beam experiment. This may be associated with the actuators in the latter being slightly thinner than those in the plate experiment. This strengthens the assumption of uncoupled transducer and substrate dynamics in the beam experiment.

The correlation between the experimental and theoretical results in the plate experiment for frequency response is weaker than that obtained in previous experiments conducted by the authors ${ }^{21-23}$ for monolithic piezoelectric transducers. This is partly due to the use of thicker Aluminum plates $(3.15 \mathrm{~mm}$ versus $1 \mathrm{~mm}$ in the present work) in those experiments while maintaining the same transducer thickness $(0.3 \mathrm{~mm})$ used here. Due to this, the assumption of uncoupled transducer and plate dynamics was stronger in Refs. 21-23 than in the present test. These indicate that for thinner Aluminum plates with relatively thick MFCs, models that are more detailed might be needed for better accuracy in predicting the exact location of the peaks and their relative amplitudes. It would be desirable to use 


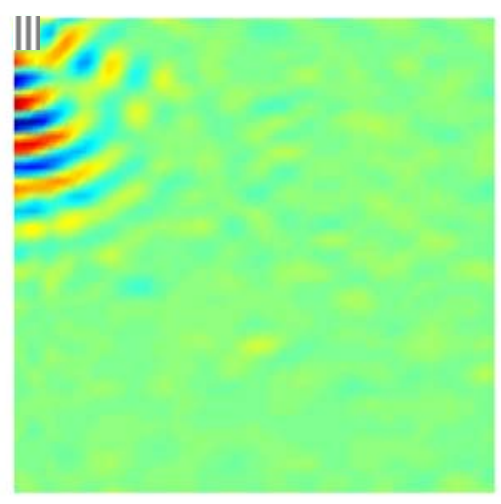

$t=100 \mu \mathrm{s}$

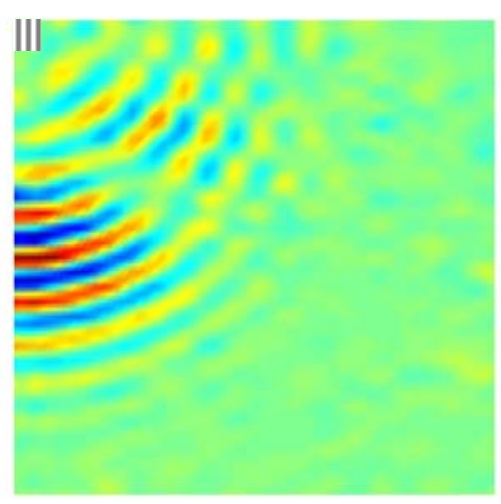

$t=150 \mu \mathrm{s}$

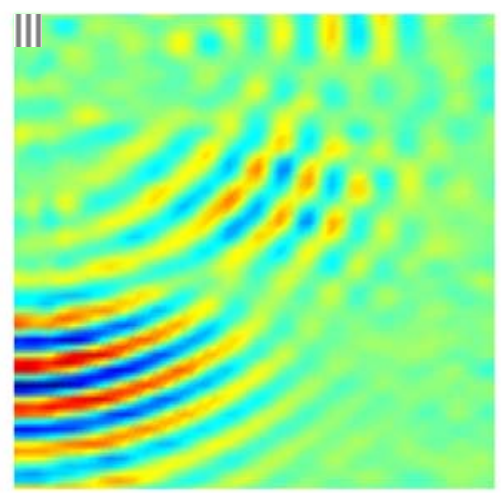

$t=200 \mu \mathrm{s}$
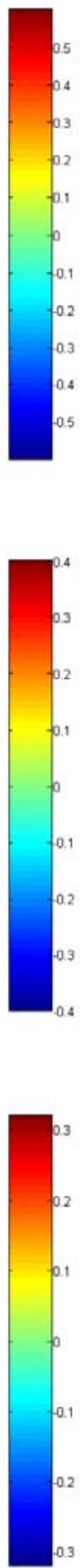

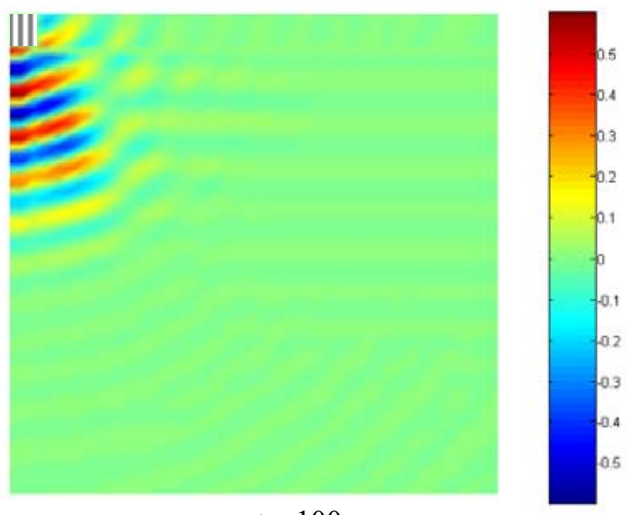

$t=100 \mu \mathrm{s}$
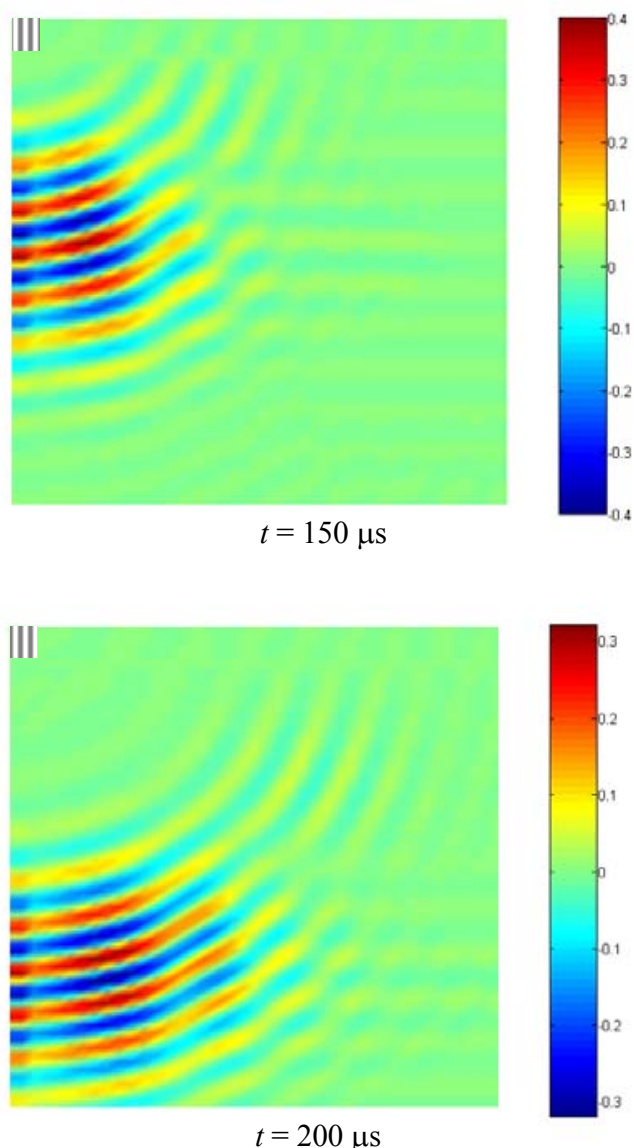

Fig. 16. Normalized surface plots showing out-of-plane velocity signals over a quarter section of the plate spanning $20 \mathrm{~cm} \times 20 \mathrm{~cm}$. The MFC is at the upper left corner, shown using a striped rectangle, and its fiber direction is along the vertical direction. a) (left) Experimental plots obtained using laser vibrometry and b) (right)

Theoretical plots obtained using the model developed in this work.

coupled models involving dynamics of both the transducer and the underlying substrate for both excitation and sensing. Since only few 3-D elasticity/piezoelectricity solutions for solids bounded in all dimensions (in this case, the actuator) can be found in the literature, such models may become intractable for purely analytical solution with no simplifying assumptions. Therefore, a hybrid approach incorporating the analytical model in this work along with some numerical formulation or experimentally determined parameters to model the actuator dynamics might be 
required. Some incipient efforts along these lines for GW excitation using monolithic piezoelectric transducers can be found in Refs. 30-31.

Another source of error comes from the impossibility of exciting a pure mode. While there were two actuators bonded on either free surface at the center of the plate, there would always be some mismatch in their piezoelectric properties due to manufacturing imperfections. In addition, due to the finite thickness of the sensor, when the wave packet is incident on it a small portion of the incident GW mode is converted to other modes due to scattering. Because of this, it was verified that some excitation of antisymmetric modes existed in the symmetric mode experiments and vice versa. An effort was made to ensure that the time window over which the peak was recorded (using the theoretical time-domain waveforms) was for the relevant mode of interest. In spite of this, the results are slightly affected by the overlapping of the two modes over certain frequency ranges.

Finally, in the beam experiment, due to the proximity of the sensor to the boundary, some of the low frequency data for the first transmitted pulse is slightly compromised by reflections from the boundary. At lower frequencies, due to the larger time-spread of the excitation signal, the reflection tends to overlap with the first transmitted pulse. This is more significant for the $\mathrm{S}_{0}$ mode due to the higher wavespeeds at low frequency. These effects were significantly reduced in the plate experiment by bonding the sensor closer to the actuators.

\section{B. Laser Vibrometer Experiment}

The experimental surface out-of-plane velocity images obtained for the plate in Fig. 16a are in good agreement with their theoretical counterparts. The theoretically predicted focused nature of the GW field along the MFC fiber direction is well captured in the experiment. There is also qualitative agreement in the patterns of the weak radiation along the other directions. However, the amplitude for those is slightly stronger in the experimental plots. In addition, the tendency of the GW field towards a directionally dependent circular crested field in the far field, which was also predicted theoretically, is evident in the experiment. There is some noise in the experimental plots, despite the use of wavelet denoising. This is because the plate, in spite of lightly sanding its surface, was a poor diffuse reflector in some areas when the laser was incident at an angle. At such points, this was partially compensated by adjusting the focus of the laser's lens. Another minor source of error in correlation is the presence of the MFC sensor (on the other free surface of the plate relative to that being scanned). This may have caused weak scattering of the GW field due to the slight change in local stiffness and mass induced by it.

\section{Concluding Remarks}

In this work, 3-D elasticity models to describe the generation of guided waves (GWs) by 3-3 anisotropic piezocomposite transducers (APTs), such as macro fiber composite (MFC) and active fiber composite transducers, in various structural configurations were presented. The configurations explored were plane Lamb-wave fields in beams with rectangular cross-section, axisymmetric GW fields in cylinders and 3-D GW fields in plates. A generic formulation to model the behavior of a 3-3 APT as GW sensor was also proposed. The use of these transducers for GW structural health monitoring was discussed. Their advantages over conventional piezoelectric wafer transducers in terms of flexibility, curved surface conformability, power efficiency and ability to excite and sense unidirectional GW fields along their fiber direction were highlighted. In the developed models, the transducer and substrate dynamics were assumed uncoupled. When used as actuator, the 3-3 APT was modeled as causing shear traction at the edges of the active area, along their fiber direction. When used as sensor, the 3-3 APT was modeled as being sensitive to the average in-plane extensional strain over its active area, again along its fiber direction. Experimental tests were conducted to verify the model's frequency response function capability for beams and plates. The results were in agreement with the theoretical predictions for the beam model, where thinner MFCs were used. For the plate model, relatively thicker MFCs were used. The trends were predicted well but there was some error in the locations of the peaks and their relative amplitudes. The sources of error were discussed. Models that account for the coupled transducer and substrate dynamics might yield better agreement with the results from the plate experiment for frequency response. In addition, the out-of-plane GW velocity patterns from MFC actuators were examined in the same plate specimen using a laser vibrometer. These patterns correlated well with the theoretical predictions. The MFC's theoretically predicted focusing ability along its fiber direction was confirmed. In the future, experiments with thicker plates are planned to examine the validity of the uncoupled actuator-substrate model for higher plate-toactuator thicknesses. Eventually, extensions of these models for composite structures will also be explored.

\section{Acknowledgments}

The authors gratefully acknowledge Prof. Karl Grosh, Mr. Robert Littrell, and Mr. Kevin King (Vibrations and Acoustics Laboratory, the University of Michigan) for providing the equipment and assisting with the laser 
vibrometer tests. This work is supported by the Space Vehicle Technology Institute under grant NCC3-989 jointly funded by NASA and DoD within the NASA Constellation University Institutes Project, with Ms. Claudia Meyer as the project manager.

\section{References}

1 Raghavan A. and Cesnik C.E.S., "Lamb wave methods in structural health monitoring," book chapter in Damage Prognosis, edited by Inman D., Farrar C.R., Lopes V. Jr. and Steffen V. Jr., John Wiley \& Sons , UK, 2005, pp. 235-257

2 Wilkie W.K., High J., and Bockman J., "Reliability testing of NASA piezocomposite actuators," Actuators 2002 Proceedings of the 8th International Conference on New Actuators, Bremen, Germany, June 10-12, 2002

3 Monkhouse R.S.C., Wilcox P.D., Lowe M.S.J., Dalton R.P., and Cawley P., "The rapid monitoring of structures using interdigital Lamb wave transducers," Smart Materials and Structures, Vol. 9, 2000, pp. 304-309

4 Badcock R.A. and Birt E.A., "The use of 0-3 piezocomposite embedded Lamb wave sensors for detection of damage in advanced fibre composites," Smart Materials and Structures, Vol. 9, 2000, pp. 291-297

5 Hayward G., Hailu B., Farlow R., Gachagan A., and McNab A., "The design of embedded transducers for structural health monitoring applications," Proceedings of the SPIE, Vol. 4327, 2001, pp. 312-323

6 Bent, A., Hagood, N., and Rodgers, J., "Anisotropic actuation with piezoelectric fiber composites," Journal of Intelligent Material Systems and Structures, Vol. 6, May 1995, pp. 338-349

7 Schulz M.J., Sundaresan M.J., Ghoshal A., and Pai P.F., "Active fiber composites for structural health monitoring," Proceedings of the SPIE, Vol. 3992, 2000, pp. 13-24

8 Datta S., Kirikera G.R., Schulz M.J., Sundaresan M.J., “Active fiber composite continuous sensors," Proceedings of the SPIE, Vol. 5062, 2003, pp. 669-676

9 Barbezat M., Brunner A.J., Huber C. and Flüeler P., "Integrated active fibre composite elements: characterization for acoustic emission and acousto-ultrasonics," Proceedings of the $15^{\text {th }}$ International Conference on Adaptive Structures and Technologies, Bar Harbor, Maine, USA, October 2004

10 Wait J. R., Park G., Sohn H. and Farrar C.R., "Plate damage identification using active wave propagation and impedance methods," Proceedings of the SPIE, Vol. 5394, 2004, pp. 53-65

11 Lamb H., "On waves in an elastic plate," Proceedings of the Royal Society of London Series A, Vol. 93, No. 651, 1917, pp. 293-312

12 Gazis D.C., "Exact analysis of the plane-strain vibrations of thick-walled hollow cylinders," Journal of the Acoustical Society of America, Vol. 30, 1958, pp. 786-794

13 Viktorov I.A., Rayleigh and Lamb waves, Plenum Press, New York, 1967

14 Ditri J. and Rose J.L., "Excitation of guided waves in generally anisotropic layers using finite sources," Journal of Applied Mechanics (Transactions of the ASME), Vol. 61, No. 2, 1994, pp. 330-338

15 Santosa F. and Pao Y.-H., "Transient axially asymmetric response of an elastic plate," Wave Motion II, Vol. 11, 1989, pp. 271-295

16 Wilcox P., "Modeling the excitation of Lamb and SH waves by point and line sources," Review of Quantitative Nondestructive Evaluation, Vol. 23, eds. Thompson D.O. and Chimenti D.E., 2004, pp. 206-213

17 Giurgiutiu V., "Lamb wave generation with piezoelectric wafer active sensors for structural health monitoring," Proceedings of the SPIE, Vol. 5056, 2003, pp. 111-122

18 Lin X. and Yuan F. G., "Diagnostic Lamb waves in an integrated piezoelectric sensor/actuator plate: analytical and experimental studies," Smart Materials and Structures, Vol. 10, 2001, pp. 907-913

19 Rose L.R.F. and Wang C.H., "Mindlin plate theory for damage detection: Source solutions," Journal of the Acoustical Society of America, Vol. 116, No. 1, 2004, pp. 154-171

20 Veidt M., Liu T. and Kitipornchai S., "Flexural waves transmitted by rectangular piezoceramic transducers," Smart Materials and Structures, Vol. 10, No. 4, 2001, pp. 681-688

21 Raghavan A. and Cesnik C.E.S., "Modeling of piezoelectric-based Lamb wave generation and sensing for structural health monitoring," Proceedings of the SPIE Symposium on Smart Structures \& Materials/ NDE 2004, Paper 5391-42, San Diego, California, March 2004

22 Raghavan A. and Cesnik C.E.S., "Piezoelectric-actuator excited-wave field solutions for guided-wave structural health monitoring," Proceedings of the SPIE Symposium on Smart Structures \& Materials/ NDE, Paper 5765-34, San Diego, California, March 2005

23 Raghavan A. and Cesnik C.E.S., "Finite dimensional piezoelectric transducer modeling for guided wave based structural health monitoring," Smart Materials and Structures, Vol. 14, 2005, pp. 1448-1461

24 González A. and Alemany C., "Determination of the frequency dependence of characteristic constants in lossy piezoelectric materials," Journal of Physics D: Applied Physics, Vol. 29, No. 9, 1996, pp. 2476-2482

25 Kastrzhitskaya E.V. and Meleshko V.V., "Propagation of harmonic waves in an elastic rectangular waveguide," Soviet Applied Mechanics (English Translation of Prikladnaya Mekhanika), Vol. 26, No. 8, Feb 1991, pp. 773-781

26 Pavlakovic B. and Lowe M., Disperse software manual Version 2.0.1 6B, Imperial College, London, UK, 2003

27 Miklowitz J., The theory of elastic waves and waveguides, North Holland, New York, USA, 1978 
28 Graff K.F., Wave motion in elastic solids, Dover Publications, New York,1991

29 "IEEE Standard on Piezoelectricity ANSI/IEEE Std 176-1987," The Institute of Electrical and Electronics Engineers Inc., New York, 1988

30 Moulin E., Assaad J. and Delebarre C., "Modeling of Lamb waves generated by integrated transducers in composite plates using a coupled finite element-normal modes expansion method," Journal of the Acoustical Society of America, Vol. 107, No. 1, 2000, pp. 87-94

31 Veidt M., Liu T. and Kitipornchai S., "Experimental investigation of the acousto-ultrasonic transfer characteristics of adhesively bonded piezoceramic transducers," Smart Materials and Structures, Vol. 9, No. 1, 2000, pp. 19-23 